\title{
RESONANCES IN THE ELECTROMAGNETIC SCATTERING BY VERY LARGE FINITE-PERIODIC GRIDS OF CIRCULAR DIELECTRIC WIRES
}

\author{
Natarov Denys M. ${ }^{1}$, Benson Trevor M. ${ }^{2}$, Altintas Ayhan ${ }^{3}$, Sauleau Ronan ${ }^{4}$, Nosich Alexander I. ${ }^{1,5}$ \\ ${ }^{1}$ Institute of Radio-Physics and Electronics NASU, vul. Proskury 12, Kharkiv, 61085, Ukraine \\ den.natarov@gmail.com \\ ${ }^{2}$ George Green Institute for Electromagnetics Research, University of Nottingham, NG7 2RD, UK \\ ${ }^{3}$ Bilkent University, Ankara 06800, Turkey \\ ${ }^{4}$ IETR, Universite de Rennes 1, Rennes Cedex 35042, France \\ ${ }^{5}$ Universite Europeenne de Bretagne, c/o IETR, Universite de Rennes 1, Rennes Cedex 35042, France
}

Diffraction of plane waves by infinite gratings is a classical research topic in the scattering theory. Using the Floquet theorem, one can reduce the infinite grating problem to the one-period problem. A characteristic feature of infinite-grating scattering is the drastic transformation of the scattering pattern and reflectance intensity if, in the process of changing the frequency or the angle of incidence, one of the Floquet harmonics is "passing over horizon." This phenomenon was first explained by Rayleigh [1] who studied theoretically the "anomalies" discovered experimentally by Wood [2]. In the simplest case of the normal incidence, these Rayleigh-Wood anomalies are observed if the period of the grating is multiple to the wavelength.

In contrast, when solving a problem of diffraction by a finite grating, for example, by a grid of $M$ wires, one cannot reduce it to one period and must treat the grid as an $M$-body scatterer [3]. It is quite interesting to find out what is the effect of periodicity on the scattering by finite grids, and how large the number $M$ should be to consider the grid as "physically infinite." As a criterion, one can choose the appearance of sharp lobes in the far-field scattering pattern in the directions of the Floquet-modes of infinite counterpart; however it is difficult to quantify this effect. Still besides of the Rayleigh anomaly, among other Wood anomalies in the infinite-grating scattering one can see the resonances on the "grating modes," whose frequencies are just below the Rayleigh frequencies [4]. Therefore in this paper we study the formation of such resonance in the scattering of the Hpolarized plane waves by the large enough finite grids of sub-wavelength dielectric wires.

Finite grid of equidistantly located parallel wires

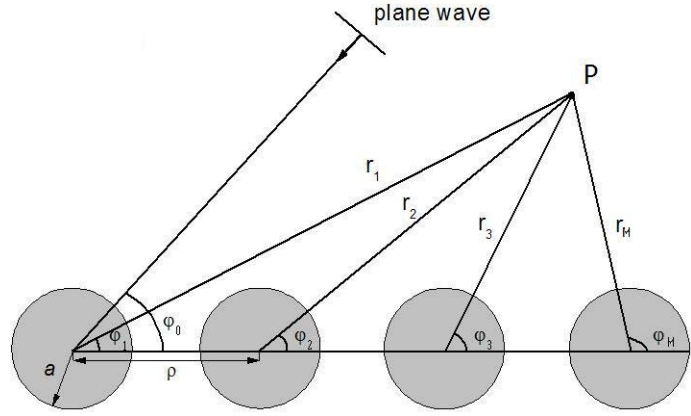

Fig. 1. Scattering geometry and notations is shown in Fig. 1. The wires are modeled as infinite circular cylinders with the same radius $a$ and relative dielectric permittivity $\varepsilon$. The distance between adjacent cylinders is $\rho$ and their number is $M$. The global coordinates have the origin at the center of the first cylinder. For a 2-D problem, a scalar function $U$, which represents either $E_{z}$ or $H_{z}$ scattered-field component, must satisfy the Helmholtz equation, $\left(\Delta+k_{ \pm}^{2}\right) U^{ \pm}(\vec{r})=0$, where $k_{-}=\sqrt{\varepsilon} k_{0}$ and $k_{+}=k_{0}$ inside and outside of each cylinder, the total-field tangential components continuity conditions, the radiation condition at infinity and the condition of the local power finiteness.

The solution can be obtained by expanding the field function in terms of the azimuth exponents in the local coordinates (Fig. 1), using addition theorems for cylindrical functions, and applying the boundary conditions on the surface of all $M$ cylinders. The unknown coefficients related to the $q$-th cylinder include the effect of all interactions between the cylinders $[3,5]$. They are finally reduced to an infinite matrix equation, $(I+U) X=U^{0}$, where: $U=\left\{\delta_{i j} U^{(i, j)}\right\}_{i, j=1, \ldots M}, X=\left(x_{m}^{(1)}, \ldots x_{m}^{(M)}\right)$, $U^{0}=\left(U_{m}^{0(1)} . . U_{m}^{0(M)}\right), i, j=1, \ldots . M, m=0, \pm 1, \pm 2, \ldots$. This is an $M \times M$ block-type Fredholm second kind equation, where each block is infinite. Therefore the solution of corresponding counterpart equation with each block truncated to finite order $N$ converges when the number $N$ gets greater. Before performing systematic calculations, we have done several tests of our code and got a good agreement with numerical data published in [6-8]. 
The most important far-field scattering characteristics are the total scattering cross section (TCS), backward or radar scattering cross section (RCS), and absorption scattering cross section (ACS). The corresponding formulas are $\sigma_{s}=2 /(\pi k) \int_{0}^{2 \pi}|\Phi(\varphi)|^{2} d \varphi, \sigma_{b}=4 / k \cdot\left|\Phi\left(\varphi_{0}\right)\right|^{2}$, and $\sigma_{a}=\operatorname{Im} \varepsilon k \int_{D}|\vec{E}| d \vec{r}$ where $D$ is the totality of all cylinder inner domains. Note that ACS can be also calculated from the power conservation law (a.k.a. optical theorem): $\sigma_{a}=-4 / k \cdot \operatorname{Re} \Phi\left(\varphi_{0}+\pi\right)-2 /(\pi k) \int_{0}^{2 \pi}|\Phi(\varphi)|^{2} d \varphi$.

In computations, we have considered finite grids of parallel GaAs nanowires (refractive index is $n=\operatorname{Re} \varepsilon^{1 / 2}=3.374$ ) with radii about tens of nanometers illuminated by the plane H-polarized wave in the visible band. In this case the wavelength is larger than the radius of cylinder. Numerical results are presented in Figs. 2 to 4 . In Figs. $2 \mathrm{a}$ and 2b, we show the TCS wavelength dependences for the grids of lossless and lossy sub-wavelength nanowires, respectively. The radius of each cylinder is $a=25 \mathrm{~nm}$ and the distance between their centers (i.e. period) is $\rho=450 \mathrm{~nm}$, while the incident plane wave comes from the broadside direction, $\varphi_{0}=\pi / 2$. Note that TCS and ASC are normalized by the number of wires in the grid, $M$.

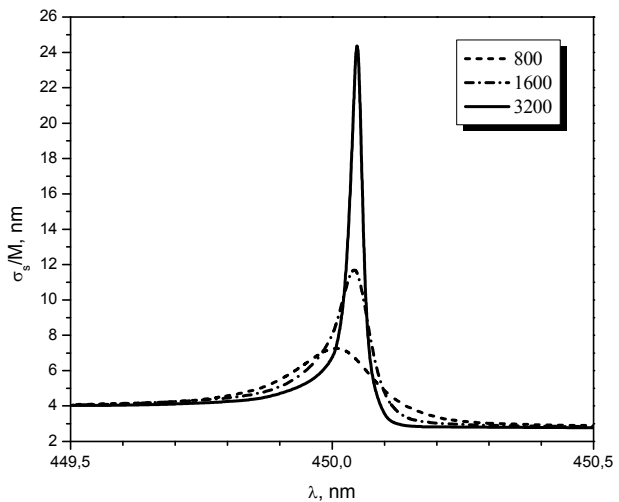

(a)

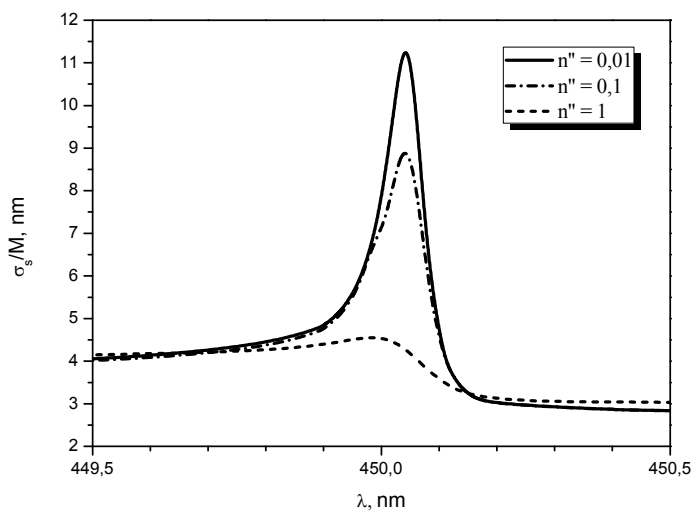

(b)

Fig. 2. Total scattering cross sections for the different grids of lossless cylinders of GaAs (a), and the same for the grid of 1600 lossy cylinders with different imaginary parts of refractive index (b).

One can see that the resonant scattering on the grating mode, i.e. at the wavelength equal to the grid period, becomes visible if the number of wires has the order of $M=1000$ or larger. As a figure of merit, one can use the Q-factor of this resonance. For the grid of 3200 lossless nanowires of GaAs it reaches 100. As follows from Fig. 2b, the presence of losses spoils the resonance and kills it completely if $n "=1$ or larger. In Figs. 3a and $3 \mathrm{~b}$, presented are the wavelength dependences of ACS and RCS, respectively, for the grids with $1600 \mathrm{GaAs}$ nanowires having complex refractive indices. Here, RCS is normalized by the grid length, $d=(M-1) \rho+2 a$.

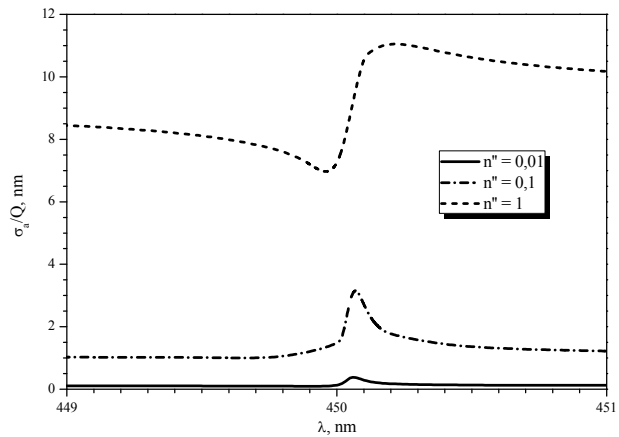

(a)

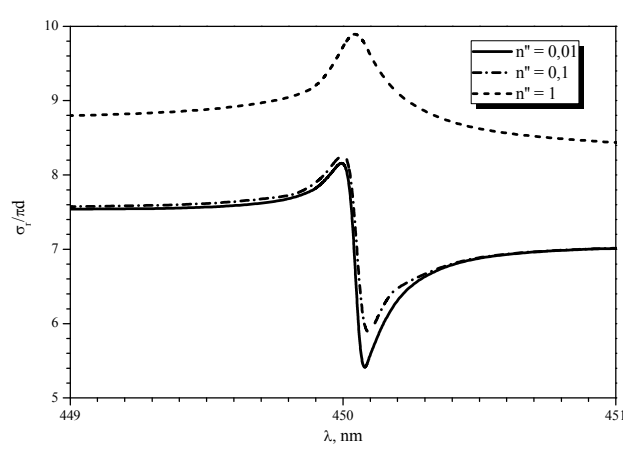

(b)

Figure 3. The same as in Fig. 2b for the absorption (a) and radar (b) scattering cross sections.

Note that As expected, both ASC and RCS increase if the losses are greater. All scattering cross sections have their value leaps in the vicinity of the resonance wavelength $450 \mathrm{~nm}$, which corresponds to the distance between the grid element centers. 
In Fig. 4, presented are the far-field scattering patterns for the grids of 1600 nanowires at the resonance wavelength, $\lambda=450.04 \mathrm{~nm}$ (Fig. $4 \mathrm{a}$ ) and at the non-resonant wavelength, $\lambda=400 \mathrm{~nm}$ (Fig. $4 \mathrm{~b}$ ). It has quite characteristic shape with very narrow main lobes looking in $\varphi=\pi / 2$ (specular reflection lobe) and $3 \pi / 2$ (shadow lobe) directions. Scattering along the grazing directions $(\varphi=0, \pi)$ is also significant because at this wavelength the \pm 1 -st Floquet harmonics of the infinite grid "pass over horizon." In the non-resonant case, the scattering pattern has all side lobes smaller however still displays sharp lobes in the mentioned two directions because the full grid length is very large, $d \approx 1600 \lambda$. It is interesting that pattern in Fig. $4 \mathrm{~b}$ has four sharp lobes corresponding to the \pm 1 -st Floquet harmonics in the infinite grid, because in this case wavelength $(400 \mathrm{~nm})$ is smaller than grating period $(450 \mathrm{~nm})$.

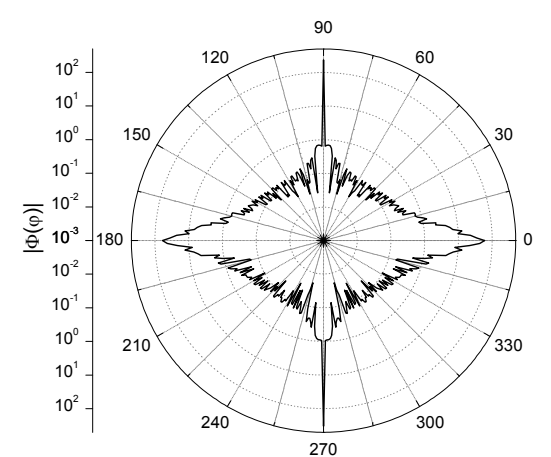

(a)

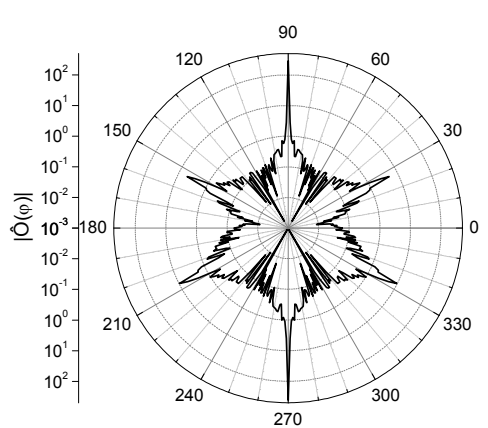

(b)

Figure 4. Far-field scattering pattern for 1600 cylinders with radius $25 \mathrm{~nm}$ and $\rho=450 \mathrm{~nm}$ at the resonance wavelength, $\lambda=450.04 \mathrm{~nm}-$ (a), at the non-resonance wavelength, $\lambda=400 \mathrm{~nm}-$ (b)

Thus, electromagnetic scattering by very large periodically structured grids of sub-wavelength wires displays sharp resonances at the wavelength equal to the grid period. This is the resonance on the grid mode. This phenomenon can be viewed as the test revealing the periodical structuring of the scattering object provided that the number of elements is large enough. As the measure of the resonance is its Q-factor, it is interesting that the resonance width for a grid of $3200 \mathrm{GaAs}$ nanowires is about $0.1 \mathrm{~nm}$. One can state that to have $Q=100$ for the studied resonance, as many as 1600 dielectric elements with refractive index $n=3.374$ are needed.

The authors are grateful to E.I. Smotrova and I.V. Ivanchenko for many helpful discussions. This work has been partially supported by the Ministry of Education and Science, Ukraine by the project M/146, the Royal Society, UK via joint project IJP-2007/R1, and the TUBITAK and NASU via exchange project 106E209.

\section{References}

1. Lord Rayleigh, "On the dynamical theory of gratings”, Proc. Royal Soc. London, vol. A-79, pp. 399-416, 1907.

2. R.W. Wood, "On a remarkable case of uneven distribution of light in a diffraction grating spectrum", Philosophical Magazine, vol. 4, pp. 396-403, 1902.

3. V. Twersky, "Multiple scattering of radiation by an arbitrary planar configuration of parallel cylinders", J. Acoust. Soc. Am., vol. 24, pp. 407-414, 1952 b.

4. R. Gomez-Medina, M. Laroche, J.J. Saenz, "Extraordinary optical reflection from sub-wavelength cylinder arrays", Optics Express, vol. 14, no 9, pp. 3730-3737, 2006.

5. A. Z. Elsherbeni, "A comparative study of two-dimensional multiple scattering techniques", Radio Science, vol. 29, no 4, pp. 1023-1033, 1994.

6. E. F. Knott, J. F. Shaeffer, M. T. Tuley, "Radar cross section $2^{\text {nd }}$ ed.”, SciTech Publishing, Raleigh, 2004.

7. H. A. Ragheb, M. Hamid, "Scattering by N parallel conducting circular cylinders", Int. J. Electronics, vol. 59, no 4, pp. 407-421, 1985.

8. J. P. Kottman, O. J. F. Martin, "Plasmon resonant coupling in metallic nanowires", Optics Express, vol. 8, no 12 , pp. $655-663,2001$. 